\title{
IL-6 Inhibitors in the Treatment of Serious COVID-19: A Promising Therapy?
}

\author{
Shubham Atal ${ }^{1}$ [D $\cdot$ Zeenat Fatima $^{1}$
}

Published online: 13 June 2020

(c) Springer Nature Switzerland AG 2020

\begin{abstract}
At present, there are no proven agents for treatment of coronavirus disease (COVID-19). The available evidence has not allowed guidelines to clearly recommend any drugs outside the context of clinical trials. The novel coronavirus SARS-CoV-2 that causes COVID-19 invokes a hyperinflammatory state driven by multiple cells and mediators like interleukin (IL)-1, IL-6, IL-12, and IL-18, tumor necrosis factor alpha (TNF $\alpha$ ), etc. Considering the proven role of cytokine dysregulation in causing this hyperinflammation in the lungs with IL-6 being a key driver, particularly in seriously ill COVID-19 patients, it is crucial to further explore selective cytokine blockade with drugs like the IL-6 inhibitors tocilizumab, sarilumab, and siltuximab. These targeted monoclonal antibodies can dampen the downstream IL-6 signaling pathways, which can lead to decreased cell proliferation, differentiation, oxidative stress, exudation, and improve clinical outcomes in patients with evident features of cytokine-driven inflammation like persistent fever, dyspnea and elevated markers. Preliminary evidence has come for tocilizumab from some small studies, and interim analysis of a randomized controlled trial; the latter also being available for sarilumab. International guidelines do include IL-6 inhibitors as one of the options available for severe or critically ill patients. There has been increased interest in evaluating these drugs with a series of clinical trials being registered and conducted in different countries. The level of investigation though perhaps needs to be further intensified as there is a need to focus on therapeutic options that can prove to be 'life-saving' as the number of COVID-19 fatalities worldwide keeps increasing alarmingly. IL-6 inhibitors could be one such treatment option, with generation of more evidence and completion of a larger number of systematic studies.
\end{abstract}

\section{Introduction}

At the time of writing, there has been a total of $>5.8$ million cases of coronavirus disease (COVID-19) worldwide, and more than 0.36 million deaths; the USA being the most heavily affected followed by Brazil, Russia, UK and Spain [1]. The mortality from this pandemic has been shown to vary between $1 \%$ to more than $7 \%$ [2]. The biggest concerns are the transmissibility of this virus leading to high rates of infection as it spreads in the population at a rate of $0.8-3 \%$, higher than the normal flu. Management of serious cases where respiratory failure from pneumonia and subsequent acute respiratory distress syndrome (ARDS) arising from hyperinflammation in the lungs, is the leading cause

Shubham Atal

shubham.pharm@aiimsbhopal.edu.in

1 Department of Pharmacology, 3rd Floor, AIIMS Bhopal, AIIMS Medical College Building, Saket Nagar, Bhopal 462020, India of mortality [3, 4]. It affects men more than women, since the X-chromosomes express more genes for immunity [5].

Current management of COVID-19 is supportive and there are no complete concrete clinical trial data yet supporting any preventive or therapeutic drugs or biologics. Current management guidelines in various nations are largely relying on anecdotal evidence or evidence from a few small completed studies or very few interim analyses. Agents previously tried in Severe Acute Respiratory Syndrome (SARS) and Middle East Respiratory Syndrome (MERS), and a handful of repurposed drugs are being considered as the main potential candidates to treat COVID-19 along with approaches like convalescent plasma therapy. Oxygen therapy and ventilator support have also been an integral part of treatment protocols.

Amongst these, the antimalarial, anti-arthritis drug hydroxychloroquine had been touted as a 'game-changer drug' globally up until recently, although the initial favorable evidence had come only from small methodologically flawed French and Chinese studies [6]. Subsequently, more studies with mixed results have been published on safety and 


\section{Key Points}

There is no proven treatment for coronavirus disease (COVID-19) as of yet, and current treatment guidelines do not recommend any particular drugs outside the context of clinical trials. Sufficient clinical evidence is lacking for all drugs that are being tried and studied.

Considering the proven role of cytokine dysregulation in serious COVID-19 and interleukin (IL)-6 being the key driver of this hyperinflammation, which can cause multi-organ failure, a series of clinical trials with IL-6 inhibitors like tocilizumab, sarilumab and siltuximab are underway. Some preliminary evidence is available for their clinical efficacy.

With the increasing case fatalities, focus is needed on therapeutic options that can prove to be life saving. More extensive evidence for clinical utility of IL-6 inhibitors in serious COVID-19 should be generated by conducting exploratory and larger systematic studies.

efficacy of hydroxychloroquine. The largest amongst these is the recently published multinational registry analysis of the use of hydroxychloroquine or chloroquine with or without a macrolide for treatment of COVID-19 in more than 95,000 hospitalized patients, which reported no benefit in hospital outcomes; instead there was an association with higher mortality and an increased frequency of ventricular arrhythmias [7]. Yet countries like USA, France, Brazil, and Israel have already been importing this drug from countries like India, which is the largest generic producer of this molecule. Among the other most promising treatment options are the human immunodeficiency virus (HIV) drug combination lopinavir/ritonavir, used in different countries in treating COVID-19 on a case-by-case basis, and the investigational new broad spectrum antiviral drug remdesivir, for which US Food and Drug Administration (FDA) has granted an Emergency Use Authorization (EUA) for the treatment of hospitalized COVID-19 patients [8]. Initial positive results have been declared for this drug, especially improved time to recovery in moderate-to-severe illness, which is sure to raise a lot of hope [9]. WHO's large scale, multinational 'Solidarity' clinical trial, and the DisCoVeRy trial launched in Europe included these drugs along with beta interferon in different arms, although the WHO temporarily suspended the randomization to the hydroxychloroquine arm of its trial in view of safety concerns [10]. These pivotal trials focus on evaluating the current most promising unproven treatment options for COVID 19, mainly focusing on hospitalized patients (moderate-severe and critically ill cases). It is pertinent to note that recently released guidelines from the National Institutes of Health (NIH), the Infectious Diseases Society of America guidelines and latest WHO interim guidance on clinical management of COVID-19 state that there are no recommendations for or against any drugs per se for COVID-19, out of the context of clinical trials right now [11-13]. IL-6 inhibitors are one class of drug that initially seemed to be under the radar in these initial frontline discussions and the major trials; drugs like tocilizumab, sarilumab, siltuximab and clazakizumab. They are now considered to be one of the adjunctive immunomodulator therapies that can be useful in severe or critical COVID-19. In recent times, there has been marked interest and rapid pace of international investigation to study the role of IL-6 inhibitors as a therapy for serious COVID-19 as evidenced by the increasing number of trials registered for these drugs. But perhaps there is a case to examine them more closely and focus more attention on their use and evaluation in serious COVID-19.

\section{SARS-CoV-2: Host Cell Infection, Inflammation}

COVID-19 is an acute respiratory disease caused by a highly pathogenic novel coronavirus SARS-CoV-2. The widely reported symptoms of COVID-19 are extremely variable, ranging from minimal symptoms to significant hypoxia with ARDS and multiorgan involvement, which could be fatal. Accordingly, the cases are classified into asymptomatic, mild, moderate, severe and critical. The latter two categories can be considered as those having serious COVID-19, and include patients presenting with pneumonia, hypoxemia, ARDS along with extrapulmonary features like shock, encephalopathy, myocardial injury, heart failure, coagulation dysfunction and acute kidney injury [13].

This virus primarily attacks airway and alveolar epithelial cells, vascular endothelial cells and macrophages in the lung, which express the angiotensin-converting enzyme 2 (ACE2), which is the host target receptor for SARS-CoV-2 [14-16]. The ACE2 expression in the lung cells is downregulated, which causes acute lung injury and dysfunction of the reninangiotensin system (RAS) [17-20]. Further, there is pyroptosis (inflammatory programed cell death seen with cytopathic viruses) which, in association with vascular leakage, triggers subsequent local inflammatory response [21-23]. This involves increased secretion of the pro-inflammatory cytokines and chemokines IL- $1 \beta$, IL-6, interferon gamma (IFN $\gamma$ ), monocyte chemoattractant protein 1 (MCP1), IFN $\gamma$ induced protein 10 (IP-10), etc. into the blood [24]. In most cases, this inflammatory reaction ameliorates the pulmonary infection and the patient recovers. Unfortunately, a dysfunctional immune response occurs in some cases, referred to 
as "Cytokine Release Syndrome" (CRS), which triggers an exaggerated release of cytokines leading to widespread pulmonary inflammation and lung damage via secretion of proteases and free radical production. There is desquamation of alveolar cells, hyaline membrane formation and pulmonary edema limiting the gas exchange in the lung, leading to difficult breathing and hypoxemia, making the lung more susceptible to secondary infection $[25,26]$.

\subsection{Interleukin-6 and 'Cytokine Release Syndrome'}

IL-6 is a multifactorial cytokine and plays a central role in acute inflammation. It is produced by monocytes and macrophages stimulated by Toll-like receptors, which in turn stimulate various cell populations. IL- $1 \beta$ and TNF $\alpha$ are the main activators of IL-6 expression [27]. IL-6 works through different signal transduction pathways [28]. In the classical pathway, it binds to both transmembrane and soluble form of its receptor IL-6R; the complex binds to the membrane protein gp130 and downstream signaling and gene expression are triggered. In the trans pathway, the complex of sIL- 6 with the receptor binds to gp130, and intracellular signal transduction is initiated. In the next step, the JAK-STAT, RAS-RAF and other pathways are activated, promoting cellular proliferation, differentiation, oxidative stress, and immune regulation. The classical IL-6 signal is limited to the cells (macrophages, neutrophils, T cells, etc.) that express IL-6R. But when IL-6 levels increase, which is what happens in subsets of patients with COVID-19, the signal is widely expressed due to the ubiquitous nature of gp130. Drugs like tocilizumab bind with cell-related and the soluble IL-6R, thus inhibiting both classical and trans signals.

"Cytokine Release Syndrome" or CRS is a phenomenon thought to be implicated in serious COVID-19 as evidenced by multiple studies, there is an uncontrolled release of cytokines like IL-1, IL-6, IL-12, and IL-18, $\mathrm{TNF} \alpha, \mathrm{IFN} \gamma$, and other inflammatory mediators, which in turn may increase alveolar-capillary gas exchange, thereby reducing oxygenation in the pulmonary tissue. This phenomenon is thought to be part of the underlying pathology for many conditions such as ARDS, sepsis, Graft-versus-Host Disease (GvHD), primary and secondary hemophagocytic lymphohistiocytosis (HLH) and complication of CAR-T cell therapies (for which IL-6 inhibitor therapy is approved) [28]. Higher blood plasma levels of IL-2, IL-7, IL-10, granulocyte colony-stimulating factor (G-CSF), IP-10, MCP1, macrophage inflammatory protein $1 \alpha(\mathrm{MIP} 1 \alpha)$ and TNF have been observed in severe COVID19 patients requiring intensive care [24]. However, IL-6 is the key driver of this hyperinflammatory process in COVID-19. Unremitting fever, hyperferritinemia and significantly elevated IL-6, similar to that seen in secondary hemophagocytic lymphohistiocytosis (sHLH), have been consistently reported in studies [3, 29]. Evidence from a meta-analysis of 6 studies revealed 2.9-fold higher mean IL-6 concentrations in patients with complicated COVID-19 than non-complicated disease [30]. The involvement of exaggerated host immune response is also suggested by findings that ARDS occurs in some SARS patients despite decreasing viral load. Furthermore, tissue necrosis and interstitial macrophages and monocyte infiltrations in the lungs, heart and gastrointestinal mucosa have been demonstrated in postmortem pathology in COVID-19 patients [25, 31]. IL-6 inhibitors thus have potential to be effective in such serious cases of COVID19 infections by preventing the severe damage to lung tissue caused by the CRS [32]. Figure 1 shows the effect of viral infection on various cells and the possible role of cytokine blockade through drugs like IL-6 inhibitors.

\section{IL-6 Inhibitors in COVID-19: Preliminary Findings and Implications}

Published preclinical data regarding efficacy of IL-6 inhibitors against SARS-CoV2 are not readily available. However, previous evidence from preclinical studies on SARS-CoV has demonstrated that inhibiting nuclear factor kappa-B (NF- $\mathrm{kB}$ ), a key transcription factor of IL-6, or infecting animals with SARS-CoV lacking the coronavirus envelope (E) protein, which is a strong stimulus to NF- $\mathrm{KB}$ signaling, increased animal survival and resulted in reduced IL-6 levels [33].

IL-6 inhibitors are categorized under immune-based therapies, separate from the antiviral therapies [11]. Evidence from previous pandemics of SARS and MERS reported no survival benefits with corticosteroids; in fact delayed viral clearance was observed from the respiratory tract and blood with high rates of complications including hyperglycemia, psychosis, and avascular necrosis with use of corticosteroids [34]. Hence, corticosteroids are generally reserved for COVID-19 in specific situations like refractory septic shock $[11,35]$. However, selective immunosuppression is likely to be beneficial in the COVID-19-associated states of pulmonary hyperinflammation. The targeted monoclonal antibodies against IL- 6 can selectively dampen this process and improve clinical outcomes in patients with evident features of cytokine driven inflammation like persistent fever, dyspnea, and elevated inflammatory markers. A further rationale has been provided by research indicating that viral load is associated with elevated IL-6 levels in severely ill COVID19 patients [36]. It has been proposed that early diagnosis of CRS in COVID-19 patients through screening tools like the 
Fig. 1 Cytokine release with coronavirus disease (COVID19) infection and possible role of IL-6 inhibitors. IL interleukin, INF $\gamma$ interferon gamma, $T N F \alpha$ tumor necrosis factor alpha
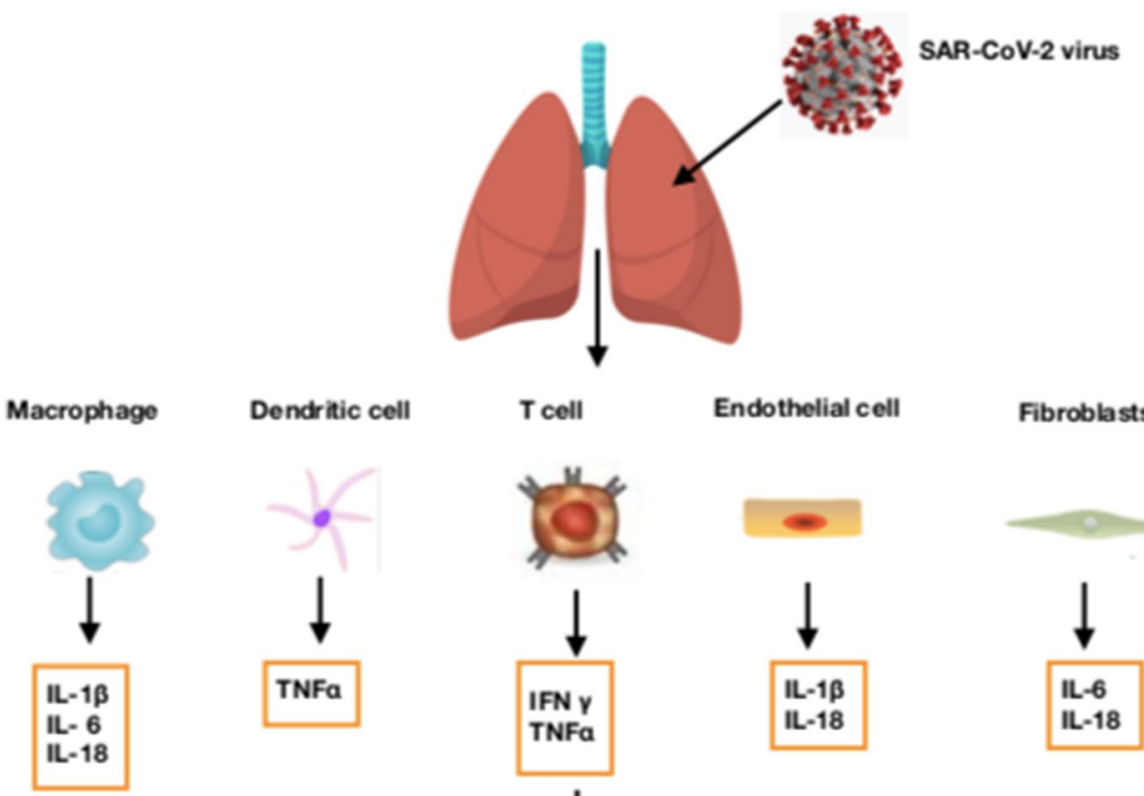

T coll

Endothelial cell

Fibroblasts
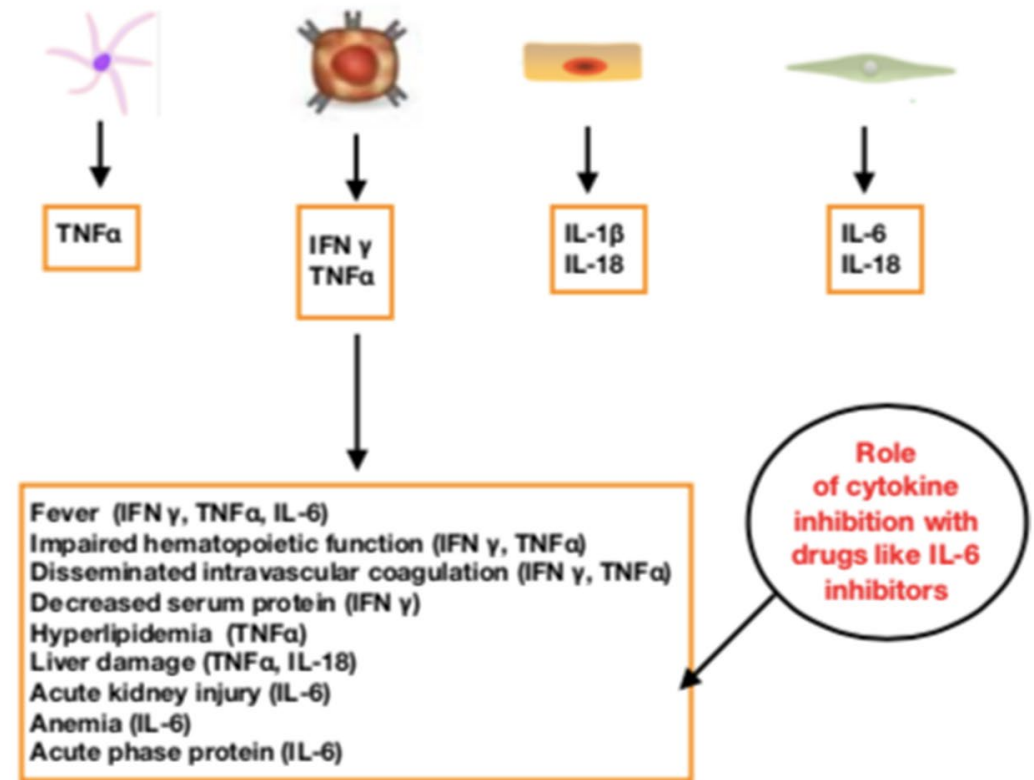

H-score used for HLH, and prompt immunomodulation with IL-6 inhibitors may be beneficial, as in HLH [37]. A recent consensus published by international pulmonologists supported the consideration of these drugs in critical illnessrespiratory failure, shock, multiorgan involvement [38]. China's National Health Commission (NHC) also included tocilizumab in its 7 th updated diagnosis and treatment plan for COVID-19 2020 [39]. They are also mentioned in the $\mathrm{NIH}$ treatment guidelines and the WHO interim guidance, both of which cite insufficient clinical data to recommend either for or against the use of these drugs — the same as for other drugs [11, 13].

Tocilizumab (Actemra) and sarilumab (Kevzara) are antagonists of the IL-6 receptors, with FDA approval to treat moderate-to-severe rheumatoid arthritis not responding to disease-modifying anti-rheumatic drugs (DMARDs), the former also approved for giant cell arteritis, polyarticular juvenile idiopathic arthritis, systemic juvenile idiopathic arthritis, and CAR-T cell-induced CRS [40, 41]. Another FDA-approved IL-6 receptor antagonist is siltuximab (Sylvant), which is indicated for the treatment of patients with multicentric Castleman's disease (MCD) who are HIV negative and human herpesvirus-8 (HHV-8) negative [42]. Preliminary evidence of efficacy in COVID-19 is available for tocilizumab through small studies. One study in severe COVID-19 patients showed successful outcomes in 21 patients, with most receiving only one dose of tocilizumab $400 \mathrm{mg}$ along with standard of care, including lopinavir and methylprednisolone. It showed improved clinical outcomes in $91 \%$ of patients in terms of improvement in lung function and successful discharge with a mean of 15.5 days; however, lack of a comparator group and blinding were important limitations [6]. Further, a retrospective observational study in 15 COVID-19 patients reported a good response in patients treated with tocilizumab and recommend repeated doses of tocilizumab in critically ill COVID-19 patients [43]. However, the number of cases reported was again small and the duration of treatment studied might not have been sufficient. 


\section{Clinical Trials with IL-6 Inhibitors}

Several randomized controlled trials or 'RCTs' (in excess of 50 in number) of tocilizumab, sarilumab and siltuximab, alone or in combination, are now proposed in patients with severe COVID-19, and are underway mainly in China, Western Europe, USA, Russia, Malaysia, and Australia [44-46].

Some of the early initiated studies include a multicenter RCT of tocilizumab alone in patients with COVID-19 pneumonia and elevated IL-6 in China (ChiCTR2000029765) targeting 188 patients [47] and another Chinese trial on 150 patients assessing tocilizumab in combination with favipiravir, a broad spectrum anti-viral agent (ChiCTR2000030894, NCT04310228) [48].

Genentech, the innovator company of tocilizumab, has also initiated a randomized, double-blind, placebo-controlled Phase III clinical trial (COVACTA, NCT04320615) to evaluate the safety and efficacy of tocilizumab plus standard of care in hospitalized adult patients with severe COVID-19 pneumonia compared with placebo plus standard of care; it aims to recruit approximately 330 patients globally [49]. The primary and secondary endpoints of the study include clinical status, mortality, mechanical ventilation, and ICU variables.

In Italy, a single-arm Phase II trial (NCT04317092) and a parallel observational cohort study, is enrolling patients with COVID-19 pneumonia in Naples to study efficacy of a single dose of tocilizumab $8 \mathrm{mg} / \mathrm{kg}$ [50]. This study has two primary outcome measures: arrest in deterioration of pulmonary function, and improvement in pulmonary function.

Another Italian Phase II open-label trial (NCT04315480) with tocilizumab $8 \mathrm{mg} / \mathrm{kg}$ single dose is being conducted in patients with severe multifocal interstitial pneumonia due to COVID-19 to evaluate its role in the virus-induced cytokine storm, in blocking deterioration of lung function or even promoting a rapid improvement of clinical conditions, preventing naso-tracheal intubation and/or death [51].

In Denmark, there is an open-label, multicenter sequential and cluster randomized trial (TOCIVID, NCT04322773) to compare the effect of either one of three IL-6 inhibitor administrations (i.e. intravenous tocilizumab, subcutaneous tocilizumab, and subcutaneous sarilumab), relative to the standard of care, in patients with severe SARS-CoV-2 pneumonia [52].

In France, an open-label Phase II RCT (CORIMUNOTOCI, NCT04331808) is being conducted by the public assistance-hospitals of Paris. Although unpublished yet, the interim results of this study were released recently through a press release. Sixty-five of the 129 patients were randomized to receive tocilizumab $8 \mathrm{mg} / \mathrm{kg}$ (1-2 injections) along with standard of care, and 64 received standard of care alone. A significantly lower proportion of the patients in tocilizumab arm attained the primary outcome of need for ventilation or death at Day 14 [53-55].

As for all other potential COVID-19 treatments, more evidence is desired at this point of time and further rigorous clinical studies are warranted.

For sarilumab, a Phase II/III trial of the intravenous formulation has been initiated in March 2020 by Sanofi and Regeneron, for hospitalized patients with severe COVID19 (NCT04315298) in USA, Italy, Spain, Germany, France, Japan, Canada and Russia [56]. The double-blind trial has an adaptive design with two parts and is anticipated to enroll up to 400 patients. The first part is recruiting patients across multiple sites in the USA and will evaluate the effect of sarilumab on fever and need for supplemental oxygen. The second, larger, part of the trial will evaluate improvement in longer-term outcomes, including prevention of mortality and reduction in need for mechanical ventilation, supplemental oxygen, and/or hospitalization. The early results from this study seem to show that its utility may be reserved for the 'critically' ill patients [57]. Another openlabel randomized Phase II/III clinical trial (CORIMUNOSARI, NCT04324073) with sarilumab in COVID-19 patients with moderate, severe or critical pneumonia aims to compare the outcomes of sarilumab-treated patients with those receiving outcomes of standard of care as well as with patients being treated with other immunomodulators [58]. More studies have since been registered or initiated for assessing sarilumab.

For siltuximab a Phase II, randomized, open-label study to compare the efficacy and safety of siltuximab versus methylprednisolone in hospitalized patients with COVID 19 pneumonia has begun recruiting patients in Spain (NCT04329650) [46]. A retrospective observational case-control study evaluating the use of siltuximab in patients diagnosed with COVID-19 infection who have developed serious respiratory complications is also registered in Italy (NCT04322188) [46].

\section{Discussion}

SAR-CoV-2 infects the human airway, vascular and immune cells, and may cause serious damage to the respiratory tract, particularly the lungs. The severity of the disease depends on the efficiency of the immune system [5]. The data so far available indicate that the viral infection can lead to an excessive immune reaction in the host in some cases so severe to be labelled as CRS, which is a hyperinflammatory state.

SAR-CoV-2 is primarily attacked by immune cells including mast cells (MCs), present in the respiratory tract and in the nasal cavity [59]. When the virus infects the respiratory 
tract, it causes a respiratory syndrome with consequent release of cytokines like interleukin IL-1 and IL-6. These in turn are mediators of further lung inflammation, fever, and fibrosis [60]. Suppression of these pro-inflammatory cytokines has been beneficial therapeutically in many inflammatory conditions, including viral infections. Considering the proven role of CRS in serious COVID-19, early identification of hyperinflammation, and its management using existing, approved therapies such as steroids, intravenous immunoglobulins, selective cytokine inhibitors should be done to prevent mortality in serious COVID-19, has been recommended [32].

Whilst the currently ongoing multiple trials for IL-6 inhibitors will answer important questions regarding the clinical usefulness of this class of drugs, the number of these studies is still proportionately lower than the clinical trials underway or announced for drugs like hydroxycholorquine. This is despite the fact that most of the recently published data for this drug have not been encouraging. A retrospective analysis of 368 patients hospitalized with confirmed COVID-19 infection in all US Veterans Health Administration medical centers during one month, showed no evidence of benefit with hydroxychloroquine [61]. Similar disappointing results and opposing conclusions have also been obtained in other studies $[62,63]$. In fact, safety concerns related to the use of hydroxychloroquine or chloroquine in COVID-19 patients had been incited by a recently published large observational analysis, in response to which the WHO temporarily suspended the hydroxychloroquine arm within the Solidarity trial while the safety data was reviewed by the Data Safety Monitoring Board; the hydroxycholorquine arm has now been reinstated and the registry analysis retracted since $[7,10]$. This overwhelming fascination with hydroxychloroquine until recently has continued across the globe from USA, Europe to China and India, perhaps due to the drug being inexpensive? Currently, remdesivir has been proven to be successful treatment for COVID-19 with the announcement of its encouraging results [9]. But it has to be borne in mind that this is still an investigational agent, with safety and long-term effects being much less explored; the global availability also remains a contentious issue, although the innovator company has entered into collaboration with multiple local manufacturers in key regions of the world.

With the dangerously rising death toll of this pandemic, it is very pertinent to look at treatment options which can literally 'save lives' among the critically ill serious COVID-19 patients. Multiple new manifestations of COVID-19 are now being recognized ranging from gastrointestinal symptoms to neurological dysfunctions, including stroke, but the predominant cause of death still remains respiratory failure, which to a large extent is due to the hypoxemic effect of the virus with an accompanying hyperinflammation in many patients.
This is where the IL-6 inhibitors can play a crucial role. The repurposed use of these FDA-approved agents may score over the highly promising investigational new drug such as Gilead's remdesivir, due to widespread experience with their clinical use in rheumatological conditions, and their known acute as well as long-term safety profile. Their off-label use may also be relatively more feasible across the globe due to ready availability with production and distribution capabilities being already in place.

Safety could actually be one of the main deterrents in the use of these drugs. Serious risk of infections has been listed as a boxed warning, although this is seen mainly with concomitant use of immunosuppressants or corticosteroids. To prevent the secondary infections, prophylactic antibiotics may be indicated. The drugs are known to cause hepatic, endocrinological, metabolic, hematological, gastrointestinal, cardiovascular adverse effects in varying frequencies [40-42]. Similar safety concerns exist for all other so-called promising treatment options for COVID-19 at the moment, albeit in differing frequencies and severity. The risk-benefit ratio has to be constantly assessed, between attenuation of life-threatening hyperinflammation and serious risk of adverse effects.

At a time when reducing mortality due to COVID-19 is unquestionably one of the primary objectives of management, these drugs may prove to be a life-saving adjunctive treatment option on the basis of their mechanism of action in serious or critical cases where chosen to be used judiciously. A significant barrier that may be holding them back from being used or tested as extensively as some of the other options is probably the very high cost of these monoclonal antibodies, which are under patent protection by the innovator companies. The Average Wholesale Price (AWP) in the USA, as available on the web, for $400 \mathrm{mg}$ tocilizumab and $200 \mathrm{mg}$ sarilumab, $400 \mathrm{mg}$ is $\$ 2765$ and $\$ 1831.29$, respectively [6].

Although there are numerous RCTs currently underway to evaluate tocilizumab, sarilumab and siltuximab, more urgency and focus is deserved by these drugs due to their potential in preventing mortality in serious COVID-19. There are still countries in the world that are significantly affected by COVID-19, where very few ongoing studies on IL-6 inhibitors can be found. Mechanical ventilators, which have been considered the last resort in the treatment protocol of critically ill patients, have high costs and availability issues. A growing body of evidence is also indicating that they may not be very useful in preventing mortality in serious patients, with multiple reports suggesting overuse of ventilators without much benefit and increase in harm [64].

There is perhaps a need to divert some of those resources to procure these drugs for wider use in eligible critically ill patients. The innovator companies can also contribute 
during these desperate times for humanity, by making these molecules available at lowest possible prices, thus allowing more off-label use of these drugs, thereby generating more preliminary anecdotal and scientific evidence.

\section{Conclusions}

To conclude, it seems that in the race for a successful antiviral treatment for COVID-19, anti-inflammatory, immunomodulatory approaches for the serious patients are now being increasingly explored, but more evidence remains to be generated. The IL- 6 inhibitor drugs belong to this category of treatment options, available at hand, yet whose potential has perhaps not been fully assessed and understood, with clinical trials still limited to a few countries. With the strong rationale for their use in serious or critically ill COVID-19 patients with predominant pulmonary involvement, and features of hyperinflammation, it may be very useful to widely explore IL-6 inhibitors as treatment options in clinical settings for serious COVID-19, and have more rigorous systematic evaluation through scientific studies.

In the fight against the highly transmissible and deadly SARS-CoV-2 causing COVID-19, these drugs can help save lives if used judiciously in appropriate cases.

Author contributions Both the authors have contributed to the conception, literature review, drafting, review and finalization of the work.

\section{Compliance with Ethical Standards}

Funding None.

Conflict of interest None.

\section{References}

1. COVID-19 Dashboard by the Center for Systems Science and Engineering (CSSE) at Johns Hopkins University (JHU). Available at: https://coronavirus.jhu.edu/map.html. Accessed 28 May 2020.

2. Onder G, Rezza G, Brusaferro S. Case-fatality rate and characteristics of patients dying in relation to COVID-19 in Italy [JAMA Network]. Available at: https://jamanetwork.com/journals/jama/ fullarticle/2763667. Accessed 29 Apr 2020.

3. Ruan Q, Yang K, Wang W, Jiang L, Song J. Clinical predictors of mortality due to COVID-19 based on an analysis of data of 150 patients from Wuhan, China. Intensive Care Med [Epub ahead of print]. 2020;1-4. Available at: https://www.ncbi.nlm.nih.gov/pmc/ articles/PMC7131986/. Accessed 29 Apr 2020.

4. Conti P, Gallenga CE, Tetè G, Caraffa A, Ronconi G, Younes A, et al. How to reduce the likelihood of coronavirus-19 (CoV19 or SARS-CoV-2) infection and lung inflammation mediated by IL-1. J Biol Regul Homeost Agents [Epub ahead of print]. 2020;34(2). Available at: https://pubmed.ncbi.nlm.nih. gov/32228825/?from_term $=$ Caraffa+A\&from_cauthor_id=32228 $825 \&$ from_pos $=1$. Accessed 29 May 2020.

5. Conti P, Younes A. Coronavirus COV-19/SARS-CoV-2 affects women less than men: clinical response to viral infection. J Biol Regul Homeost Agents [Epub ahead of print]. 2020;34(2). Available at: https://www.biolifesas.org/biolife/2020/04/07/coronaviru s-cov-19-sars-cov-2-affects-women-less-than-men-clinical-respo nse-to-viral-infection/. Accessed 29 May 2020.

6. Cennimo DJ, Bergman SJ. Coronavirus Disease 2019 (COVID19) Treatment \& Management. Updated. 2020. [Medscape] Available at: https://emedicine.medscape.com/article/2500114-treat ment\#d14. Accessed 6 May 2020.

7. Mehra MR, Desai SS, Ruschitzka F, Patel AN. Hydroxychloroquine or chloroquine with or without a macrolide for treatment of COVID-19: a multinational registry analysis. Lancet [Epub ahead of print] 2020. Available at: https://doi.org/10.1016/S0140 -6736(20)31180-6. Accessed 24 May 2020.

8. Remdesivir EUA letter of authorization. Available at: https:// www.fda.gov/media/137564/download. Accessed 8 May 2020.

9. Beigel JH, Tomashek KM, Dodd LE, Mehta AK, Zingman BS, Kalil AC, et al. Remdesivir for the treatment of COVID-19-a preliminary report. N Engl J Med. 2020. https://doi.org/10.1056/ NEJMoa2007764.

10. WHO Director-General' opening remarks at the media briefing on COVID-19. 2020. [WHO website]. Available at: https://www. who.int/dg/speeches/detail/who-director-general-s-opening-remar ks-at-the-media-briefing-on-covid-19---25-may-2020. Accessed 29 May 2020.

11. National Institutes of Health. Coronavirus disease 2019 (COVID19) treatment guidelines. 2020. [NIH website]. Available at: https ://www.covid19treatmentguidelines.nih.gov/. Accessed 24 Apr 2020.

12. Infectious Diseases Society of America. Infectious Diseases Society of America guidelines on the treatment and management of patients with COVID-19. 2020. [IDS society website]. Available at: https://www.idsociety.org/practice-guideline/covid-19-guide line-treatment-and-management/. Accessed 24 Apr 2020.

13. Clinical management of COVID-19 INTERIM GUIDANCE. Available at: https://apps.who.int/iris/bitstream/handle/10665 1332196/WHO-2019-nCoV-clinical-2020.5-eng.pdf?seque nce $=1$ \&isAllowed $=y$. Accessed 28 May 2020.

14. Jia HP, Look DC, Shi L, Hickey M, Pewe L, Netland J, et al. ACE2 receptor expression and severe acute respiratory syndrome coronavirus infection depend on differentiation of human airway epithelia. J Virol. 2005;79(23):14614-21.

15. Xu H, Zhong L, Deng J, Peng J, Dan H, Zeng X, et al. High expression of ACE2 receptor of 2019-nCoV on the epithelial cells of oral mucosa. Int J Oral Sci. 2020. https://doi.org/10.1038/s4136 8-020-0074-x.

16. Hamming I, Timens W, Bulthuis ML, Lely AT, Navis G, van Goor H. Tissue distribution of ACE2 protein, the functional receptor for SARS coronavirus. A first step in understanding SARS pathogenesis. J Pathol. 2004;203(2):631-7.

17. Imai Y, Kuba K, Rao S, Huan Y, Guo F, Guan B, et al. Angiotensin-converting enzyme 2 protects from severe acute lung failure. Nature. 2005;436(7047):112-6.

18. Imai Y, Kuba K, Penninger JM. The discovery of angiotensinconverting enzyme 2 and its role in acute lung injury in mice. Exp Physiol. 2008;93(5):543-8.

19. Kuba K, Imai Y, Rao S, Gao H, Guo F, Guan B, et al. A crucial role of angiotensin converting enzyme 2 (ACE2) in SARS coronavirus-induced lung injury. Nat Med. 2005;11(8):875-9.

20. Kuba K, Imai Y, Penninger JM. Angiotensin-converting enzyme 2 in lung diseases. Curr Opin Pharmacol. 2006;6(3):271-6. 
21. Zhang H, Zhou P, Wei Y, Yue H, Wang Y, Hu M, et al. Histopathologic changes and SARS-CoV-2 immunostaining in the lung of a patient with COVID-19. Ann Intern Med. 2020;172(9):629-32.

22. Chen IY, Moriyama M, Chang MF, Ichinohe T. Severe acute respiratory syndrome coronavirus viroporin 3 a activates the NLRP3 inflammasome. Front Microbiol. 2019. https://doi.org/10.3389/ fmicb.2019.00050.

23. Yang M. Cell pyroptosis, a potential pathogenic mechanism of 2019-nCoV infection. SSRN. 2020. https://doi.org/10.2139/ ssrn. 3527420 .

24. Huang C, Wang Y, Li X, Ren L, Zhao J, Hu Y, et al. Clinical features of patients infected with 2019 novel coronavirus in Wuhan. China Lancet. 2020;395:497-506.

25. Xu Z, Shi L, Wang Y, Zhang J, Huang L, Zhang C, et al. Pathological findings of COVID-19 associated with acute respiratory distress syndrome. Lancet Respir Med. 2020;8:420-2.

26. Tian S, Hu W, Niu L, Liu H, Xu H, Xiao SY, et al. Pulmonary pathology of early phase 2019 novel coronavirus (COVID-19) pneumonia in two patients with lung cancer. J Thorac Oncol. 2020. https://doi.org/10.1016/j.jtho.2020.02.010.

27. Hunter CA, Jones SA. IL-6 as a keystone cytokine in health and disease. Nat Immunol. 2015;16(5):448-57.

28. Liu B, Li M, Zhou Z, Guan X, Xiang Y. Can we use interleukin-6 (IL-6) blockade for coronavirus disease 2019 (COVID-19)-induced cytokine release syndrome (CRS)? J Autoimmun [Epub ahead of print]. 2020. Accessed 25 Apr 2020.

29. Zhou F, Yu T, Du R, Fan G, Liu Y, Liu Z, et al. Clinical course and risk factors for mortality of adult inpatients with COVID19 in Wuhan, China: a retrospective cohort study. Lancet. 2020;395:1054-62.

30. Coomes EA, Haghbayan H. Interleukin-6 in COVID-19: a systematic review and meta-analysis. medRxiv. 2020. Available at: https ://doi.org/10.1101/2020.03.30.20048058. Accessed 29 Apr 2020.

31. Yao XH, Li TY, He ZC, Ping YF, Liu HW, Yu SC. A pathological report of three COVID-19 cases by minimally invasive autopsies. Zhonghua Bing Li Xue Za Zhi. 2020;49:411-7.

32. Mehta P, Mc Auley DF, Brown M, Sanchez E, Tattersall RS, Manson JJ, et al. COVID-19: consider cytokine storm syndromes and immunosuppression. Lancet. 2020;395:1033-4.

33. DeDiego ML, Nieto-Torres JL, Regla-Nava JA, JimenezGuardeno JM, Fernandez-Delgado R, Fett C, et al. Inhibition of NF-kappaB-mediated inflammation in severe acute respiratory syndrome coronavirus-infected mice increases survival. J Virol. 2014;88:913-24.

34. Stockman LJ, Bellamy R, Garner P. SARS: systematic review of treatment effects. PLoS Med. 2006. https://doi.org/10.1371/ journal.pmed.0030343.

35. Russell CD, Millar JE, Baillie JK. Clinical evidence does not support corticosteroid treatment for 2019-nCoV lung injury. Lancet. 2020;395:473-5.

36. Chen X, Zhao B, Qu Y, Chen Y, Xiong J, Feng Y, et al. Detectable serum SARS-CoV-2 viral load (RNAaemia) is closely associated with drastically elevated interleukin 6 (IL-6) level in critically ill COVID-19 patients. medRxiv. 2020. Available at: https://doi.org/10.1101/2020.02.29.20029520. Accessed 29 Apr 2020.

37. Henter JI, Chow CB, Leung CW, Lau YL. Cytotoxic therapy for severe avian influenza A (H5N1) infection. Lancet. 2006;367:870-3.

38. Treatment options. In: Joseph T, Moslehi MA, editors. International Pulmonologist's Consensus On COVID-19, 2nd edn. Available at: https://www.unah.edu.hn/dmsdocument/9674-conse nso-internacional-de-neumologos-sobre-covid-19-version-ingles. Accessed 27 Apr 2020.

39. National Health Commission and State Administration of Traditional Chinese Medicine. Diagnosis and treatment protocol for novel coronavirus pneumonia. Available at: https://www.chinalawtranslate.com/wp-content/uploads/2020/03/Who-translation. pdf. Accessed 25 Apr 2020.

40. Tocilizumab: Drug information. [UpToDate website]. Available at: https://www.uptodate.com/contents/tocilizumab-drug-infor mation\#F9773765. Accessed 26 Apr 2020.

41. Sarilumab: Drug information. [UpToDate website]. Available at: https://www.uptodate.com/contents/sarilumab-drug-informatio n?source=see_link\#F50133614. Accessed 29 Apr 2020.

42. Siltuximab: Drug information. [UpToDate website]. Available at: https://www.uptodate.com/contents/siltuximab-drug-information. Accessed 28 May 2020.

43. Luo P, Liu Y, Qiu L, Liu X, Liu D, Li J. Tocilizumab treatment in COVID-19: a single center experience. J Med Virol. 2020. https ://doi.org/10.1002/jmv.25801.

44. ClinicalTrials.gov [Internet]. Available at: https://clinicaltrials. gov/ct2/results?cond=Covid-19\&term=tocilizumab\&cntry $=\&$ state $=\&$ city $=\&$ dist $=$. Accessed 28 May 2020 .

45. ClinicalTrials.gov [Internet]. Available at: https://clinicaltrials. gov/ct $2 /$ results? cond $=$ sarilumab+COVID $+19 \&$ term $=\&$ cntry $=\&$ state $=\&$ city $=\&$ dist $=$. Accessed 28 May 2020.

46. ClinicalTrials.gov [Internet]. Available at: https://clinicaltrials. gov/ct $2 /$ results? cond $=$ siltuximab + covid $+19 \&$ term $=\&$ cntry $=\&$ state $=\&$ city $=\&$ dist $=$. Accessed 28 May 2020 .

47. Chinese Clinical Trial Registry. A multicenter, randomized controlled trial for the efficacy and safety of tocilizumab in the treatment of new coronavirus pneumonia (COVID-19). Available at: http://www.chictr.org.cn/showprojen.aspx?proj=49409. Accessed 26 Apr 2020.

48. Favipiravir combined with tocilizumab in the treatment of corona virus disease 2019. Available at: https://clinicaltrials.gov/ct2/ show/NCT04310228. Accessed 8 May 2020.

49. A study to evaluate the safety and efficacy of tocilizumab in patients with severe COVID-19 pneumonia (COVACTA). Available at: https://clinicaltrials.gov/ct2/show/NCT04320615. Accessed 8 May 2020.

50. Tocilizumab in COVID-19 Pneumonia (TOCIVID-19) (TOCIVID-19). Available at: https://clinicaltrials.gov/ct2/show/ NCT04317092. Accessed 8 May 2020.

51. Tocilizumab for SARS-CoV2 (COVID-19) Severe Pneumonitis. Available at: https://clinicaltrials.gov/ct2/show/NCT04315480. Accessed 29 Apr 2020.

52. Anti-il6 treatment of serious COVID-19 disease with threatening respiratory failure (TOCIVID). Available at: https://clinicaltrials. gov/ct2/show/NCT04322773. Accessed 8 May 2020.

53. CORIMUNO-19-Tocilizumab Trial-TOCI (CORIMUNOTOCI) (CORIMUNO-TOC). Available at: https://clinicaltrials. gov/ct2/show/NCT04331808. Accessed 8 May 2020.

54. Tocilizumab improves significantly clinical outcomes of patients with moderate or severe COVID-19 pneumonia. Available at: https://pipelinereview.com/index.php/2020042874458/Antib odies/Tocilizumab-improves-significantly-clinical-outcomes-ofpatients-with-moderate-or-severe-COVID-19-pneumonia.html. Accessed 8 May 2020.

55. Press Release. First randomized study favorable to tocilizumab in Covid-19, in France. Available at: https://www.apmnews.com/ depeche/0/350489/premiere-etude-randomisee-favorable-au-tocilizumab-dans-le-covid-19\%2C-en-france. Accessed 8 May 2020.

56. Evaluation of the efficacy and safety of sarilumab in hospitalized patients with COVID-19. Available at: https://clinicaltrials.gov/ ct2/show/NCT04315298. Accessed 8 May 2020.

57. Erman M, Joseph S. Regeneron, Sanofi arthritis drug may only help critical coronavirus patients: study. Available at: https:// www.reuters.com/article/us-health-coronavirus-regeneron-pharm s/regeneron-sanofi-to-treat-only-critical-covid-19-patients-witharthritis-drug-idUSKCN2291OD. Accessed 30 Apr 2020. 
58. Cohort multiple randomized controlled trials open-label of immune modulatory drugs and other treatments in COVID-19 patients-sarilumab trial-CORIMUNO-19-SARI (CORIMUNO-SARI). Available at: https://clinicaltrials.gov/ct2/show/ study/NCT04324073. Accessed 8 May 2020.

59. Kritas SK, Ronconi G, Caraffa A, Gallenga CE, Ross R, Conti P. Mast cells contribute to coronavirus-induced inflammation: new anti-inflammatory strategy. J Biol Regul Homeost Agents [Epub ahead of print]. 2020. 10.23812/20-Editorial-Kritas.

60. Conti P, Ronconi G, Caraffa A, Gallenga CE, Ross R, Frydas I, et al. Induction of pro-inflammatory cytokines (IL-1 and IL-6) and lung inflammation by Coronavirus-19 (COVI-19 or SARSCoV-2): anti-inflammatory strategies. J Biol Regul Homeost Agents. 2020. https://doi.org/10.23812/CONTI-E.

61. Magagnoli J, Narendran S, Pereira F, Cummings T, Hardin JW, Sutton SS, et al. Outcomes of hydroxychloroquine usage in United States veterans hospitalized with Covid-19. medRxiv. 2020. Available at: https://www.medrxiv.org/conte nt/10.1101/2020.04.16.20065920v2. Accessed 30 Apr 2020.
62. Tang W, Cao Z, Han M, Wang Z, Chen J, Sun W, et al. Hydroxychloroquine in patients with COVID-19: an open-label, randomized, controlled trial. medRxiv. 2020. Available at: https:// www.medrxiv.org/content/10.1101/2020.04.10.20060558v1. Accessed 30 Apr 2020.

63. Molina JM, Delaugerre C, Le Goff J, Mela-Lima B, Ponscarme D, Goldwirt L, et al. No evidence of rapid antiviral clearance or clinical benefit with the combination of hydroxychloroquine and azithromycin in patients with severe COVID19 infection. Med Mal Infect [Epub ahead of print]. Available at: https://www.sciencedirect.com/science/article/pii/ S0399077X20300858?via\%3Dihub. Accessed 29 Apr 2020.

64. Dondorp AM, Hayat M, Aryal D, Beane A, Schultz MJ. Respiratory support in novel coronavirus disease (covid-19) patients, with a focus on resource-limited settings. Am J Trop Med Hyg. 2020;1-7. 